\title{
A Versatile Cascade of Intramolecular Vilsmeier-Haack and Azomethine Ylide 1,3-Dipolar Cycloaddition toward Tricyclic Cores of Alkaloids ${ }^{\dagger}$
}

2008

Vol. 10, No. 21

$4939-4942$

\section{François Lévesque and Guillaume Bélanger* \\ Département de Chimie, Université de Sherbrooke, 2500 boulevard Université, Sherbrooke, Québec J1K 2R1, Canada}

guillaume.belanger@usherbrooke.ca

Received August 27, 2008

\section{ABSTRACT}

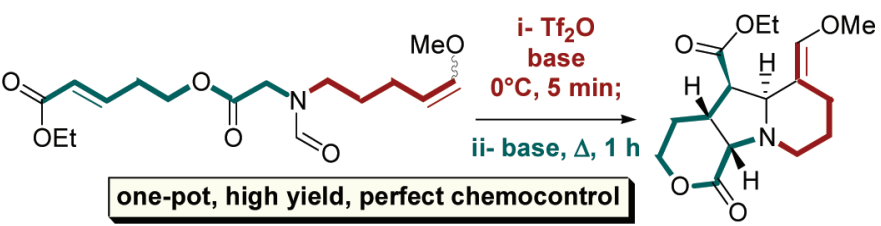

In the pursuit of synthetic efficiency, we developed an innovative one-pot transformation of linear substrates into bi- and tricyclic adducts using a cascade of amide activation, nucleophilic cyclization, azomethine ylide generation, and subsequent inter- or intramolecular 1,3-dipolar cycloaddition. Despite the high density and variety of functional groups on the substrates, the sequence occurred with perfect chemoselectivity with good to excellent yields.

The pursuit of synthetic efficiency has been driving synthetic chemists over the years. The rapid and controlled increase in molecular complexity constitutes an enormous challenge for the construction of polycyclic natural products. Reactions in cascade certainly offer a powerful means to reach this goal, as several bonds can be formed in a single operation. ${ }^{1}$ With this in mind, we became interested in exploring a new cascade of reactions that combines our recently developed Vilsmeier-Haack cyclization methodology ${ }^{2}$ with the generation of an azomethine ylide and a subsequent intramolecular 1,3-dipolar cycloaddition (Scheme 1). ${ }^{3}$

† Dedicated to Professor Pierre Deslongchamps on the occasion of his 70th birthday.

(1) (a) Tietze, L. F.; Brasche, G.; Gericke, K. M. Domino Reactions in Organic Chemistry; WILEY-VCH: Weinheim, 2006; p 672. (b) Nicolaou, K. C.; Edmonds, D. J.; Bulger, P. G. Angew. Chem., Int. Ed. 2006, 45, 7134.

(2) (a) Bélanger, G.; Larouche-Gauthier, R.; Ménard, F.; Nantel, M.; Barabé, F. Org. Lett. 2005, 7, 4431. (b) Bélanger, G.; Larouche-Gauthier, R.; Ménard, F.; Nantel, M.; Barabé, F. J. Org. Chem. 2006, 71, 704.

(3) For reviews on azomethine ylides, see: (a) Coldham, I.; Hufton, R. Chem. Rev. 2005, 105, 2765. (b) Tsuge, O.; Kanemasa, S. Adv. Heterocycl. Chem. 1989, 45, 231.

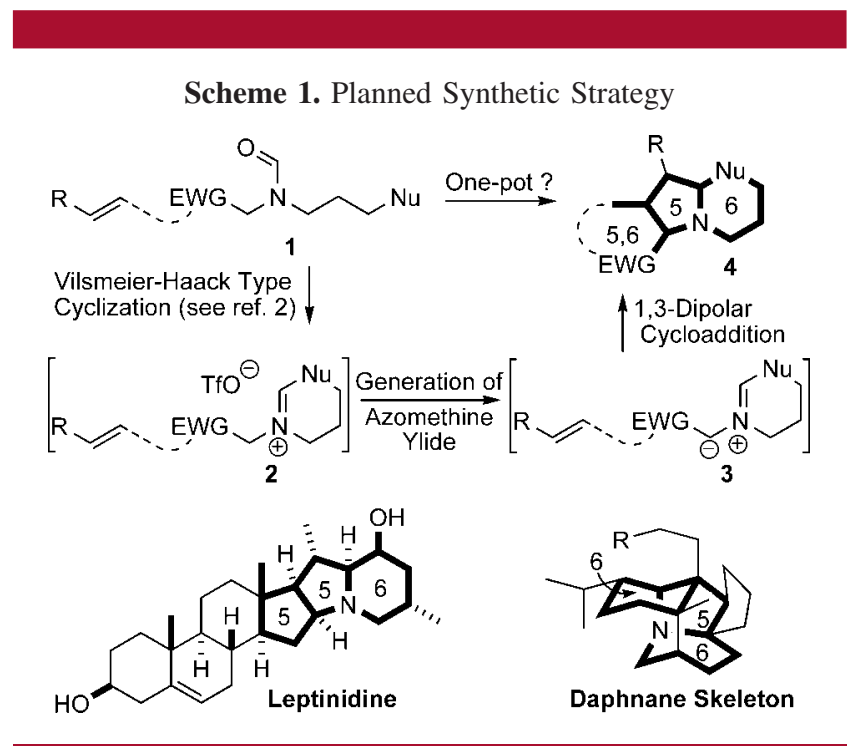

The versatility of our planned synthetic strategy is noteworthy. The branches bearing the nucleophile and the 
dipolarophile can be attached anywhere about the amide. Upon the proposed key transformation, the various possible assemblies for a starting material substrate will lead to very diverse skeletons that belong to a wide range of alkaloid families of pharmaceutical interest.

We already demonstrated that iminium ions (cf. 2), generated from intramolecular Vilsmeier-Haack reactions, are stable and could sometimes be isolated. ${ }^{2}$ Addition of a suitable tethered dipolarophile should trap the ylide $\mathbf{3}$, itself formed through a deprotonation of $\mathbf{2}$, and generate the second and third cycles of the desired cascade product 4 . Such structures are found in numerous steroidal alkaloids, such as leptinidine ${ }^{4}$ daphnane alkaloids, and others. The greatest challenge in the development of this reaction sequence is certainly the numerous chemoselectivity issues associated with each step of the desired cascade since the reactivity and the order of reaction of the four required functional groups must be perfectly controlled.

In order to prove the viability of our planned synthetic strategy, we prepared and then tested a first model compound bearing a methyl enol ether as the nucleophile for the Vilsmeier-Haack cyclization and a tethered dipolarophile for the final 1,3-dipolar cycloaddition. Preparation of precursor 9 started with iodination of known alcohol $\mathbf{5}^{5}$ (Scheme 2). Iodide 6 then served in the alkylation of formamide to

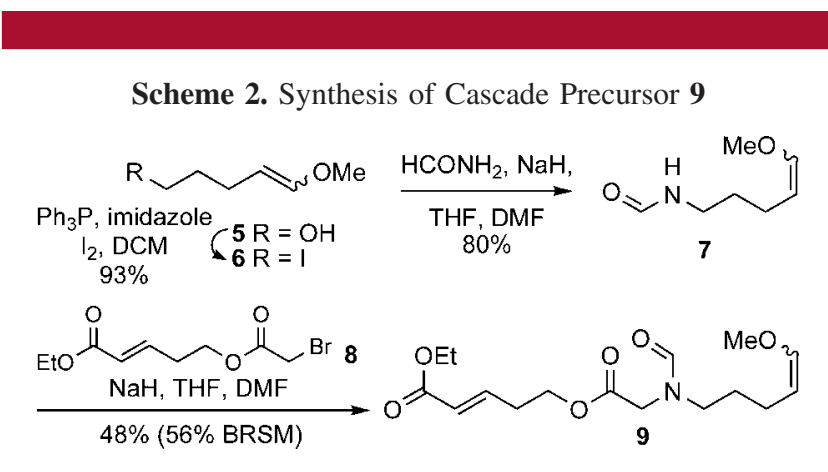

generate 7. The latter was alkylated again, this time with known bromoester $\mathbf{8}^{6}$

The possibility of generating three cycles in a single sequence was then examined. When the highly functionalized model substrate 9 was treated with triflic anhydride in the presence of 2,6-di-tert-butyl-4-methylpyridine (DTBMP), the resulting triflyliminium ion reacted exclusively with the electron-rich alkene in a Vilsmeier-Haack-type cyclization (Scheme 3). Upon deprotonation, the azomethine ylide $\mathbf{1 0}$ was generated and then reacted with the remaining electron-poor alkene in a highly diastereoselective intramolecular 1,3-dipolar cycloaddition.

The success of the reaction hinged on ylide generation at a temperature that could then promote the dipolar cycloaddition. Lower temperatures resulted in complete decomposition of the reactive ylide. ${ }^{7}$ The whole cascade of reactions occurred with perfect chemoselectivity. An 8:1 mixture of 11/12 was obtained in $77 \%$ overall yield, which is excellent for this one-pot tricyclization from linear $9 .{ }^{8}$ The relative stereochemistry of all four adjacent stereocenters in $\mathbf{1 1}$ was confirmed by single-
Scheme 3. Cascade of Intramolecular Vilsmeier-Haack-Azomethine Ylide Generation-1,3-Dipolar Cycloaddition
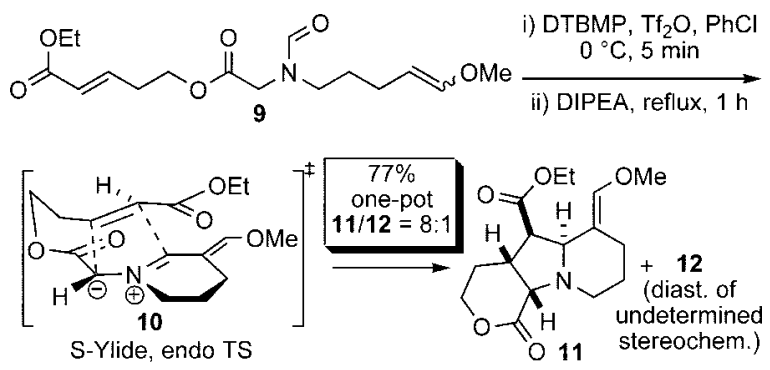

crystal X-ray analysis (Figure 1) and is the result of an endo transition state for the cycloaddition of the ylide $\mathbf{1 0}$ in an $S$ conformation; endo/exo transition state preferences and ylide conformation are discussed in detailed below.

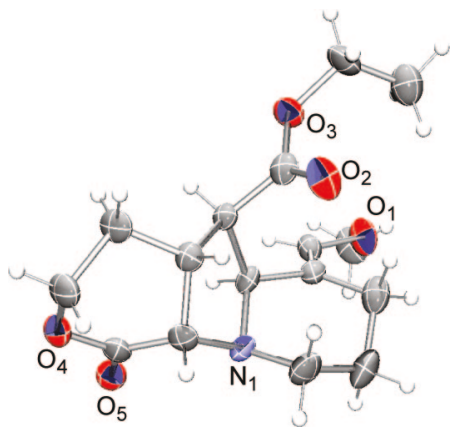

Figure 1. ORTEP representation of the single-crystal X-ray structure of $\mathbf{1 1 .}$

With this especially encouraging result, we established the feasibility of the rapid increase in molecular complexity in one operation using our synthetic strategy. For the following identification of the scope and limitations of the strategy, we opted for the study of easily accessible model compounds in a Vilsmeier-Haack cyclization-intermolecular cycloaddition sequence.

Preparation of precursors $\mathbf{1 4 b}-\mathbf{d}$ is detailed in Scheme 4. ${ }^{9}$ Acetamide was alkylated with iodide $\mathbf{6}$ to give compound 13. Alkylation of the anion of either the formamide $\mathbf{7}$ or the acetamide $\mathbf{1 3}$ with ethyl bromoacetate furnished the precursors $\mathbf{1 4 b}$ and $\mathbf{1 4 d}$, respectively. A methyl Grignard addition to $\mathbf{1 4 b}$ at low temperature produced the methylketone $\mathbf{1 4 c}$.

Since the first part of the cascade, the Vilsmeier-Haack type cyclization, has already been successfully developed, ${ }^{2}$ we concentrated our efforts on the azomethine ylide generation and the subsequent 1,3-dipolar cycloaddition. Therefore, a series of bases was screened for the azomethine ylide generation (tested with substrate 14b). Although many bases were either unreactive ${ }^{10 a}$ or caused the decomposition of the substrate, ${ }^{10 \mathrm{~b}}$ we found that DIPEA had the best balance of 
Scheme 4. Synthesis of Cascade Precursor $14 b-d$

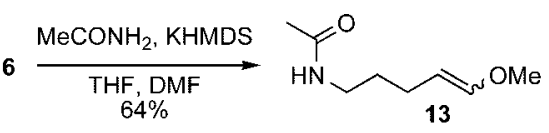

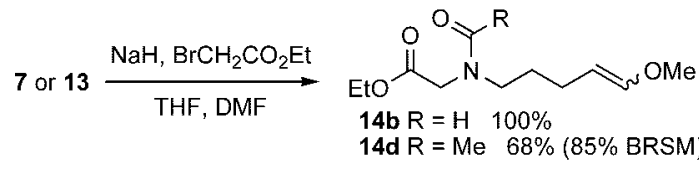

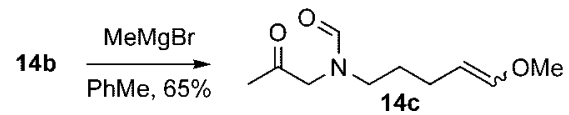

high basicity and low nucleophilicity. ${ }^{10 \mathrm{c}} \mathrm{We}$ also rapidly concluded that the azomethine ylide was unstable and needed to react immediately with a dipolarophile upon its generation. ${ }^{11}$ To this end, $\mathrm{N}$-phenylmaleimide was chosen as a prototypic highly reactive dipolarophile.

In order to study the scope of the reaction cascade, the optimized conditions were used with model compounds $\mathbf{1 4 a}-\mathbf{e}$ (Table 1). Both the silyl (14a) and methyl (14b) enol ethers led to the desired cycloadducts (entries 1 and 2, respectively), although $\mathbf{1 4 b}$ gave a much better yield (92\%), presumably because of the lower acid ${ }^{12}$ sensitivity of the methyl enol ether. Delightfully, excellent yields (82-92\%) were obtained with either the ester $(\mathbf{1 4 b}$, entry 2$)$ or the ketone (14c, entry 3). ${ }^{2,13}$

Finally, in order to verify the capacity of the reaction cascade to withstand steric hindrance, we replaced the formamide (14b, entry 2) with bulkier groups. In the case of the acetamide (14d, entry 4), no cycloadduct was observed, even though the iminium ion intermediate was completely formed. ${ }^{14} \mathrm{We}$ suspect that deprotonation leading to the resulting enamine was preferred to the generation of the ylide. ${ }^{15}$ This competing deprotonation pathway was circumvented by replacing the methyl with a phenyl (14e, entry 5). We were pleased to see that generation of a quaternary center was thus possible at this position (16e/ 17e, $63 \%$ combined yield), even though a higher cycloaddition temperature was required.

The relative stereochemistry of all cycloadducts was examined. The relationship between $\mathrm{C}_{2}$ and $\mathrm{C}_{5}$ in the cycloadducts $\mathbf{1 5}$ and $\mathbf{1 6}$ could be rationalized on the basis of the geometry of the ylide (Scheme 5).

When the substituent $\mathrm{R}^{3}$ on the amide was an hydrogen (entries 1-3), electrostatic stabilization in the $S$ conformation prevails, ${ }^{16}$ leading preferentially to $\mathbf{1 5 a}-\mathbf{c}$. Closely related examples showed that such substrates give endo and exo adducts resulting from a common $S$-ylide intermediate. ${ }^{17}$ However, $14 \mathbf{a}-\mathbf{c}$ furnished only endo adducts $\mathbf{1 5} \mathbf{a}-\mathbf{c}$ and $\mathbf{1 6 a}-\mathbf{c}$, regardless of the ylide geometry. In the case where the amide substituent $\mathrm{R}^{3}$ was a phenyl (entry 5), steric hindrance destabilized the $S$ conformation, and the cycloadducts 16e and 17e obtained both stem from a $W$-ylide, via endo and exo cycloaddition transtition states, respectively. Structures of $\mathbf{1 6 e}$ and $\mathbf{1 7 e}$ were proven by single crystal $\mathrm{X}$-Ray diffraction.

Note that in all of the cycloadducts, the enol ether had a trans geometry, as a result of the initial Vilsmeier-Haack reaction. For instance, after amide $\mathbf{1 8}$ is activated, the nucleophilic addition of the tethered enol ether gerenates a

Table 1. Scope of the Key Reaction Cascade ${ }^{a}$

entry

${ }^{a}$ Reagent and conditions: (i) DTBMP, $\mathrm{Tf}_{2} \mathrm{O}, \mathrm{CH}_{2} \mathrm{Cl}_{2}, 0{ }^{\circ} \mathrm{C}, 5 \mathrm{~min}$; (ii) $\mathrm{N}$-phenylmaleimide; (iii) DIPEA, 15 min. (b) Ratio determined by NMR analysis of crude material. (c) Combined yield of diastereoisomers. (d) Step (iii) conducted in refluxing toluene. 
Scheme 5. Geometry of the Azomethine Ylide and endo/exo Transition States

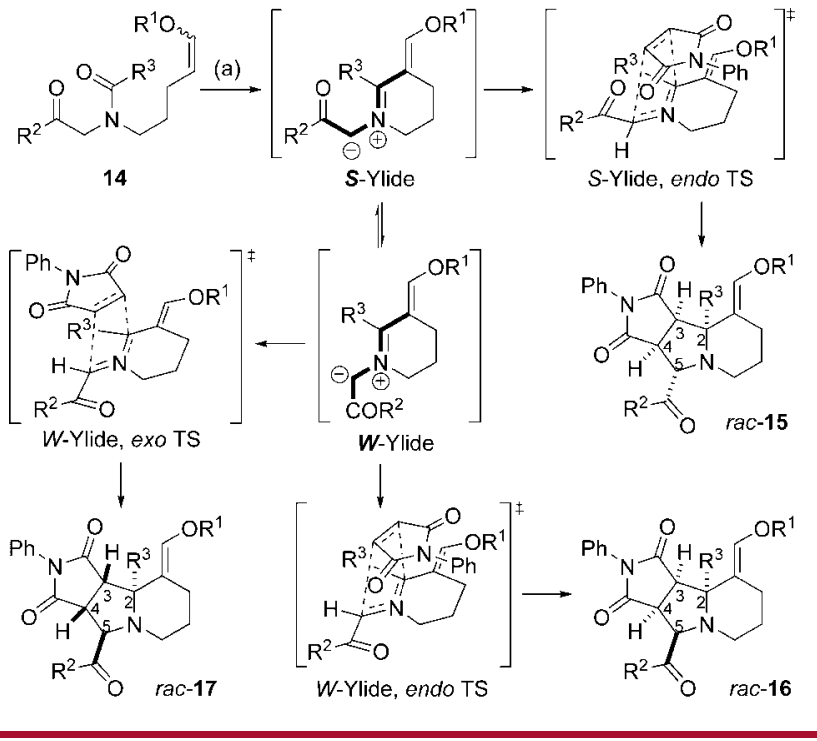

carboxonium ion 19 (Scheme 6). The latter gets deprotonated to give the less sterically congested trans enol ether 20, leading to unsaturated iminium ion $\mathbf{2 1}$ upon triflate expulsion.

In conclusion, we demonstrated that a new synthetic strategy that exploits our recently developed Vilsmeier-Haack cyclization coupled to the generation and cycloaddition of its azomethine ylide is a powerful, rapid, and efficient

(4) Coelho, R. M.; De Souza, M. C.; Sarragiotto, M. H. Phytochemistry 1998, 49, 893

(5) Methyl enol ether $\mathbf{5}$ is obtained from a Wittig olefination of $\gamma$-butyrolactol. See: Wasserman, H. H.; Cook, J. D.; Vu, C. B. J. Org. Chem. 1990, 55, 1701.

(6) Jones, R. C. F.; Howard, K. J.; Nichols, J. R.; Snaith, J. S. J. Chem. Soc., Perkin Trans. 1 1998, 13, 2061.

(7) The electron withdrawing group $\left(\mathrm{CO}_{2} \mathrm{Et}\right)$ on the dipolarophile was found to be necessary for the success of the cycloaddition. The unsubstituted terminal alkene proved to not be reactive enough, in accordance with the major $\mathrm{HOMO}_{\text {azomethine ylide }}-\mathrm{LUMO}_{\text {dipolarophile }}$ interaction in such 1,3-dipola cycloadditions. See: Houk, K. N.; Sims, J.; Watts, C. R.; Luskus, L. J. J. Am. Chem. Soc. 1968, 90, 543.

(8) The increased thermal stability of methyl enol ether over silyl enol ether was dramatic: for substrate 9, when the methyl group is substituted for a TBDMS, only $29 \%$ overall yield of tricyclic adduct was obtained.

(9) Preparation of models 14a and $\mathbf{1 4} \mathbf{e}$ is described in Supporting Information.
Scheme 6. Geometry of Enol Ether in Cycloadducts

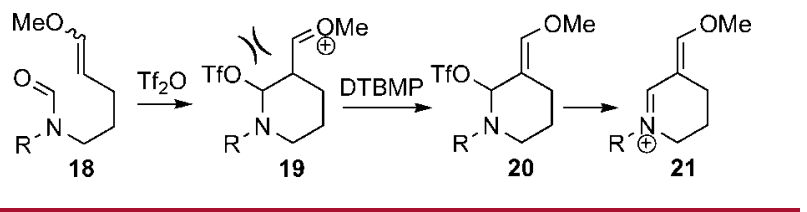

approach to polycyclic alkaloid cores. The generation of bicyclic and tricyclic adducts occurred in a single operation from an acyclic substrate with perfect chemoselectivity. Diastereoselectivity of the process is fully rationalized. Applications of our strategy to the total synthesis of alkaloids are now in progress.

Acknowledgment. This research was supported by the Natural Science and Engineering Research Council (NSERC) of Canada, FQRNT (Québec), the Canadian Fund for Innovation (CFI), and the Université de Sherbrooke. The Université de Sherbrooke is acknowledged for $\mathrm{Ph}$. D. fellowship to F.L.

Supporting Information Available: Experimental details and spectra for all new compounds, including CIF files. This material is available free of charge via the Internet at http://pubs.acs.org.

OL802010N

(10) (a) DTBMP, DABCO, $\mathrm{Cs}_{2} \mathrm{CO}_{3}$. (b) KHMDS, LTMP, and $\mathrm{NaH}$. (c) Nucleophilic bases (such as DBU) initiated undesired anionic polymerization of $\mathrm{N}$-phenylmaleimide presumably via Michael addition of the base onto $N$-phenylmaleimide.

(11) Any attempts to form the azomethine ylide prior to the addition of the dipolarophile led to low yield of cycloadduct.

(12) Compounds 15a/16a decomposed slowly upon purification on silica gel.

(13) For another example of chemoselective amide activation in the presence of a ketone, see: Barbe, G.; Charette, A. B. J. Am. Chem. Soc. 2008, 130,18 .

(14) Confirmed by ${ }^{1} \mathrm{H}$ NMR spectroscopy (see Supporting Information)

(15) Even though the resulting enamine was not observed, its formation has been proven in several occasions. For example, see: Smith, R. Livinghouse, T. Tetrahedron 1985, 41, 3559.

(16) Tsuge, O.; Kanemasa, S.; Takenaka, S. Chem. Lett. 1985, 355.

(17) Tóth, G.; Frank, J.; Bende, Z.; Weber, L.; Simon, K. J. Chem. Soc., Perkin Trans. 1 1985, 1961. 